\title{
SHAPE CHARACTERIZATION OF CONCRETE AGGREGATE
}

\author{
JiNG Hu AND PIET STROEVEN
}

Faculty of Civil Engineering and Geosciences, Delft University of Technology, Delft, The Netherlands e-mail: p.stroeven@tudelft.nl

(Accepted March 21, 2006)

\begin{abstract}
As a composite material, the performance of concrete materials can be expected to depend on the properties of the interfaces between its two major components, aggregate and cement paste. The microstructure at the interfacial transition zone (ITZ) is assumed to be different from the bulk material. In general, properties of conventional concrete have been found favoured by optimum packing density of the aggregate. Particle size is a common denominator in such studies. Size segregation in the ITZ among the binder particles in the fresh state, observed in simulation studies by concurrent algorithm-based SPACE system, additionally governs density as well as physical bonding capacity inside these shell-like zones around aggregate particles. These characteristics have been demonstrated qualitatively pertaining also after maturation of the concrete. Such properties of the ITZs have direct impact on composite properties. Despite experimental approaches revealed effects of aggregate grain shape on different features of material structure (among which density), and as a consequence on mechanical properties, it is still an underrated factor in laboratory studies, probably due to the general feeling that a suitable methodology for shape characterization is not available. A scientific argument hindering progress is the interconnected nature of size and shape. Presently, a practical problem preventing shape effects to be emphasized is the limitation of most computer simulation systems in concrete technology to spherical particles. New developments at Delft University of Technology will make it possible in the near future to generate jammed states, or other high-density fresh particle mixtures of non-spherical particles, which thereupon can be subjected to hydration algorithms. This paper will sketch the outlines of a methodological approach for shape assessment of loose (non-embedded) aggregate grains, and demonstrate its use for two types of aggregate, allowing evaluation of usefulness of the various methods.
\end{abstract}

Keywords: concrete aggregate, Fourier analysis, quantitative image analysis, shape characterisation, stereology.

\section{INTRODUCTION}

Modern technology of cementitious materials is nowadays also explicitly involved in establishing geometrical statistical features of material structure underlying material performance. The most obvious and more frequently used parameter in such approaches is material density, either applied to the particulate structure of the cement on micro-level or to that of the aggregate on meso-level. These particulate structures can be described in a more complete way by using a set of independent geometrical statistical descriptors, like number density, volume fraction, spacing, size and shape. Sieving is the established way in concrete technology for characterizing grain size in engineering applications as well as for laboratory investigations, though the inherent deficiencies have been recognized for a long time. Seen in scientific perspective, size and shape cannot be separated in an unambiguous way, of course. Still, additional methods for characterizing shape would be helpful and particularly relevant for laboratory research purposes, since shape has been found exerting significant influences on concrete performance, indeed. The conventional indirect method to determine aggregate angularity is by means of packing density measurements, which is time-consuming and labour intensive.

Shape is one of the most difficult features to define. And, without definition, quantification is impossible. Shape has been defined as "any definable combination of geometrical elements, whether real or imaginary" (Underwood, 1970). However, this does not offer a clear concept that could uniquely be interpreted for all cases occurring in practice. It is well known that the shape and surface texture of aggregate exert significant influences on workability as well as on compactability and thereby on concrete strength and durability. Hence, water (cement) content, and optimal volume fraction of fine aggregate (sand) should be adapted to shape and texture characteristics of the coarse aggregate. In the absence of quantitative information with respect to shape and surface texture, mix proportioning has become more or less a trial- 
and-error process. It would be of high research as well as of practical significance, therefore, to derive indices incorporating sufficient information on shape and surface texture of aggregates. Additionally, these indices should be obtained in a convenient and economic way so as to reduce the trial-and-error procedure involved in concrete mix proportioning as envisaged in codes and standards. This paper is therefore particularly focussing on modern methodology available for this purpose. As an illustrative example, the methodology is applied to two types of aggregates, gravel of fluvial origin and crushed rock, of which the data will be presented. Conventional image analysis techniques, stereological extrapolations, as well as Fourier analysis are employed for this purpose.

Stereology allows reliable derivation of threedimensional shape information for global characterisation of aggregates, whereas Fourier analysis can efficiently describe more detailed structure information, such as surface roughness and texture of the aggregate grains. Only a small part of the conventional shape parameters (such as roundness, circularity, elongation ratio, sphericity and flakiness) are sensitive to shape variations and can be correlated with angularity of the aggregate. As a promising alternative, Fourier coefficients can be used to describe the shape, surface roughness and texture of the aggregate grains. In addition, a new shape index is proposed on the basis of Fourier analysis, which has been demonstrated able to incorporate sufficient shape information, and can be used directly to estimate packing density of the investigated aggregate. The methodology that will be presented can be extended to other applications in concrete technology where shape characterisation of particles is at issue.

\section{CONVENTIONAL METHODS FOR SHAPE CHARACTERIZATION}

\section{EXPERIMENTAL METHODS}

The concept underlying the indirect methods is based on experimental measurements of properties of aggregate in bulk that are influenced by their shape and surface texture. Such properties include bulk density, settling velocity, rate of flow of water through gravel, behaviour of particles on an inclined surface, and the percentage voids. Angularity number suggested by Shergold (1953) is a pioneering work in the evaluation of aggregate shape using the concept of packing density (i.e., volume fraction) of aggregate. British Standards codes (BS 812, 1975) have adopted this method for the quantification of aggregate shape. However, the method has several limitations. It gives contradictory results when regular-shaped bodies are tested. Furthermore, the heavy compaction applied may cause crushing as well as artificial changes in the surface texture of the aggregate, thereby affecting the angularity number. Huang (1962) modified the concept of angularity number into a so-called Index of Aggregate Particle Shape and Texture (IAPST), which takes into consideration the voids percentage (or volume fraction) as well as the rate of change of voids percentage in mono-size aggregate under standard compaction conditions. The rate of change is supposedly dependent on the shape, angularity and surface texture of the aggregate grains. The upgraded methodology is adopted as ASTM D-3398-97 (1997) and has been used to study the influence of aggregate shape and texture on the compaction and strength characteristics of Portland cement concrete (Jamkar and Rao, 2004).

\section{QUANTITATIVE IMAGE ANALYSIS}

One major problem with image processing techniques is that only the $2 \mathrm{D}$ projection profiles of the particles can be captured and measured. The projection profiles of the aggregate grains are usually reflected by shape factors such as the aspect ratio or elongation ratio, or Feret diameter. Consequently, the third dimension, i.e., thickness of the particles is not directly obtainable from the image analysis results. Stereological theory states that area fractions (2D) of particles are unbiased estimates of volume densities (3D). Total area or area fraction of aggregate particles can be converted to mass or mass (volume) fraction by means of stereological and statistical tools, based on the assumption that aggregate particles from the same source should have more or less similar shape characteristics. The assumption is realised by incorporating a global parameter reflecting the correlation between mean thickness and measurable dimensions (e.g., breadth) of the particles.

Kwan et al. (1999) proposed a global flakiness ratio $(\lambda)$ defined as the weighed averaged of thickness to breadth of the aggregate particles. The total volume $(V)$ and the mass $(M)$ of the particles can be expressed as:

$$
\begin{gathered}
V=\sum_{i=1}^{n}(\text { thickness } \times \text { area })=\lambda \times \sum_{i=1}^{n}(\text { breadth } \times \text { area }) \\
\lambda=\frac{M / \rho}{\sum_{i=1}^{n}(\text { breadth } \times \text { area })}
\end{gathered}
$$


where $\rho$ is the volumetric density of the aggregate grains, $n$ is the number of aggregate grains in the sample. The parameter $\lambda$ calculated via Eq. 1 may serve as a flakiness indicator. The experimental investigations by Kwan et al. (1999) revealed that the parameter $\lambda$ calculated according to Eq. 1 is corresponding well to direct measurements of flakiness index. This proposal of flakiness ratio $\lambda$ will be adapted in this study for image analysis to simplify the $3 \mathrm{D}$ issue of surface shape to a $2 \mathrm{D}$ one of boundary shape of projection profiles. So, the following global shape parameters are readily derived:

$$
\begin{aligned}
& \text { Elongation ratio }=\text { Length } / \text { breadth } \\
& \text { Flakiness ratio }(\lambda)=\text { Thickness } / \text { breadth } \\
& \text { Circularity }=(\text { Perimeter })^{2} /\left(4 \pi^{*} \text { Area }\right) \\
& \text { Rectangularity }=\text { Area } /(\text { Area of bounding } \\
& \text { rectangular) }
\end{aligned}
$$$$
\text { Aspect ratio }=F_{\text {max }} / F_{\text {min }} \text {, where } F_{\text {max }} \text { and } F_{\text {min }}
$$
are maximum and minimum Feret Diameter$$
\text { Fullness }=\text { Area } /\left(F_{\max } * F_{\text {min }}\right)
$$$$
\text { Sphericity ratio }=\sqrt[3]{\lambda(\text { breadth } / \text { length })^{2}}
$$$$
\text { Shape factor }=\lambda \sqrt{\text { breadth / length }}
$$

The above-mentioned parameters are selfexplanatory. The circularity and rectangularity describe the degree of approximation of the particle profile to a circle and to a rectangle, respectively. The sphericity ratio is a $3 \mathrm{D}$ parameter depicting the similarity of the investigated particle to a sphere. However, these shape descriptors cannot regenerate the original shape of the projection profiles since they contain no information as to the boundary variations of the profiles. The study of Yamamoto et al. (2002) revealed that the conventional roundness and circularity are far from efficient descriptors of irregular geometrical shapes, i.e., not sufficiently sensitive to shape variations.

An efficient alternative is to transform the boundary variations by Fourier analysis into a set of Fourier coefficients reflecting the particle shape information. For details of the Fourier analysis, the reader is referred to, e.g., Gonzales and Woods (1992) and Beddow (1985). This contribution deals with more complicated problems of assessing the shape, lumpiness, roughness and texture of aggregate particles by stereological methods and by Fourier analysis.

\section{INNOVATIVE METHODS}

Stereological theory is readily available for global size and shape characterisations by means of 3D extrapolation of the $2 \mathrm{D}$ shape information derived from projection images. As for shape description of individual particles, an approach fundamentally different from the above mentioned shape descriptors (like roundness and circularity) in image analysis is by geometrical-statistical simulation of the shape of projection profiles. Basically, the surface of a particle is described by a parametric function of the radius (distance between the centroid to a point at the surface). Fourier analysis is a popular tool for shape characterization in this category. The advantage is that only a small number of coefficients would be sufficient to represent the shape information in a sensitive way. In other words, the Fourier coefficient series contain information on both surface roughness (smoothness) and on the local and global shape of the particles. This will allow deriving new shape parameters, which are adapted to specific issues in concrete technology, and are more efficient than the conventional ones.

\section{STEREOLOGICAL METHODS}

For single non-embedded particles, shape can be defined, but averaging for an aggregate would require doing so for volumes and surfaces of particles. Since these operations are scale dependent, this will generally not lead to manageable solutions. Most of the research on this topic has been focusing on characterizing 2D sections of embedded particles by stereological means, a situation that can be considered pertinent for cementitious materials. A special case is serial sectioning with the objective of three-dimensional reconstruction. Anatomists and biologists have extensively developed this technique for translucent samples. An alternative option is the generation of mathematically defined 2D sections or 3D surfaces as a reference for estimating shape of actual geometric elements of structure. Of the aforementioned stereological parameters, a number is particularly useful in defining shape (Table 1). For relevant literature, see Underwood (1970; 1976).

Shape factors or shape indices can be formed (and have been used) from combinations of these stereological parameters. The three-dimensional stereological parameters are related to the appropriate moments of the common particle size distribution function. This can be formulated in the following way

$$
\begin{gathered}
\bar{V}=S_{3} M_{3}(d), \bar{S}=S_{2} M_{2}(d), \\
\bar{H}_{3}=S_{1} M_{1}(d), \\
\bar{L}_{3}=S_{1}^{*} M_{1}(d), \\
\bar{k}_{S}=S_{-1} M_{-1}(d),
\end{gathered}
$$


in which $S_{3}, S_{2}, S_{1}, S^{*}{ }_{1}$ and $S_{-1}$ are dimensionless form factors, and $M_{\mathrm{n}}(d)$ is the n-th moment of the particle size distribution function.

\section{FOURIER ANALYSIS}

An alternative method of shape characterisation is to use a Fourier mathematical technique. Much research has been undertaken in the field of powder technology and geology to produce mathematical relations that may characterise the profiles of individual particles (Beddow, 1985).

Fourier analysis is an approach fundamentally different from the above mentioned shape descriptors in image analysis. The mathematical details are well described in the literature (Beddow, 1985; Gonzales and Woods, 1992). The Fourier Transform produces a complex number valued output image that can be displayed in the form of two images, either with the real and imaginary parts or with magnitude and phase. In general, only the magnitude of the Fourier Transform is displayed in image processing, as it contains most of the information of the geometric structure of the spatial domain image. Because the image in the Fourier domain is decomposed into its sinusoidal components, it is easy to examine or process certain frequencies of the image, thus influencing the geometric structure in the spatial domain. However, orientation distribution is not the subject of this study.

Fourier analysis renders possible processing the single particle azimuthal spectra for deriving shape information. Here, the distance from a boundary point to the centroid of a particle (i.e., in polar coordinates) at an azimuthal angle $\theta_{i}$ can be expanded as in Eq. 3 . Analogue to diffraction measurements, the distance can also be interpreted as intensity of signal $L_{i}$ at the angle $\theta_{i}$. In this study, 64 sampling points describe the boundary of each aggregate grain, i.e., the full range of azimuthal angles $\left(0-360^{\circ}\right)$ is divided into 64 segments (corresponding to an angular increment of $5.625^{\circ}$ ) for Fourier analysis.

$$
\begin{gathered}
R\left(\theta_{i}\right)=L_{i}=A_{0}+\sum_{n=1}^{\infty} A_{n} \cos \left(n \theta_{i}-\alpha_{n}\right), \\
\theta_{i}=5.625 i, \quad i=0,1, \ldots 63
\end{gathered}
$$

where $R\left(\theta_{i}\right)$ is the radius (i.e., distance from a boundary point to the centroid) at angel $\theta_{i}, n$ is the harmonic number, and $A_{n}$ are Fourier coefficients and $\alpha_{n}$ the phase angles for each harmonic:

$$
A_{n}=\sqrt{a_{n}^{2}+b_{n}^{2}} \quad \alpha_{n}=\tan ^{-1}\left(b_{n} / a_{n}\right) .
$$

This is generally known as Fourier method in closed form, a method that is favoured in geology research, predominantly for the analysis of roughness and textural features of granular soils (Bouman et al., 2000).

The number of sampling points (i.e., the total number of harmonics) chosen also dictates the number of descriptors gained from the Fourier analysis, and therefore the level of details described. For example, in the case of 128 sampling points, 128 possible Fourier descriptors are produced. The complex nature of Eq. 3 means that the lower order descriptors (smaller value of $n$ ) will describe the overall particle morphology and generally have the greater coefficients due to the larger described features. The values generally decay toward the descriptors at higher orders as the features become smaller, For general use as descriptors for a body of aggregate, however, only the magnitude of the descriptor coefficient is required, i.e., $A_{\mathrm{n}}$.

It should be noted that in the averaging procedure of particles within one aggregate group, the angle $\theta$ associated with radii are modified so that the radius with $\theta=0$ coincides with the longest axis. This will help to eliminate the effect of orientation distribution of the particles. In a study focusing on particle shape (as in the present case), the Fourier coefficients can be normalised by $A_{0}$ to eliminate the influences due to size variations among the investigated particles.

Table 1. Two- and three-dimensional stereological parameters relevant to shape description.

\begin{tabular}{l|l}
\hline Two-dimensional parameters & Three-dimensional parameters \\
\hline $\bar{A}$ - average area of particle sections & $\bar{V}$ - average volume of particles \\
$\bar{L}_{p}$ - average perimeter length of particle sections & $\bar{S}$ - average surface area of particles \\
$\bar{H}_{2}$ - average tangent height of particle sections & $\bar{H}_{3}$ - average tangent height of particles \\
$\bar{L}_{2}$ - average intercept length of particle sections & $\bar{L}_{3}$ - average intercept length of particles \\
$\bar{k}_{L}$ - mean curvature of particle perimeters & $\bar{k}_{S}$ - average mean curvature of particle surface \\
\hline
\end{tabular}


The set of these Fourier coefficients represents the periodical behaviour of the boundary and can be used to characterize particle shape. The lower-order number coefficients reflect the global structure of the signal variation, whereas the higher-order number coefficients do so with respect to the fine structure. In the case of 64 sampling points for each particle boundary, the Fourier coefficients $A_{1}-A_{31}$ are symmetrical to $A_{33}-A_{63}$ with $A_{32}$, i.e., $A_{31}=A_{33}$, $A_{30}=A_{34} \ldots A_{1}=A_{63}$. Hence, the profile boundary is described by 31 Fourier descriptors $\left(A_{0}\right.$ is not included). The advantage is that only a small number of coefficients are necessary to reflect the required shape information in a sensitive way. For example, a square shape is represented by a large $A_{4}$ exceeding $A_{2}$ by two orders of magnitude (with large values for $A_{4}, A_{8}, A_{12}$, etc), while an ellipse yields a large $A_{2}$ and a smaller $A_{4}$. Large values for $A_{3}, A_{6}, A_{9}$, etc, depict a triangle. The coefficients $A_{\mathrm{n}}$ are invariant features and are used in morphological analysis studies. The shape characteristics are measured on a scale of gradually resolving power. The set of Fourier coefficients can be roughly divided into three portions to describe the lumpiness, roughness and texture of the aggregate grains (Beddow, 1985):

$$
\begin{gathered}
\text { Lumpiness: } L=\sum_{n=1}^{n=8} A_{n}, \\
\text { Roughness: } R=\sum_{n=9}^{n=17} A_{n}, \\
\text { Texture: } T=\sum_{n=18}^{n=24} A_{n} .
\end{gathered}
$$

Ma et al. (2000) have investigated size and shape in powder technology by means of Fraunhofer laser diffraction measurements. Since the diffraction intensity pattern from an aperture is proportional to the Fourier transform of a transparency distribution of the aperture, a correlation coefficient curve similar to what is defined in Ma et al. (2000) has been employed in this study. For a single particle, the correlation coefficient between two azimuthal angles (with distance of $5.625^{\circ}$ in this study) is given as:

$$
\rho_{m}=\frac{1}{N} \sum_{i=1}^{N} L_{i} L_{i+m} .
$$

Different particle shapes produce a different form of this correlation coefficient curve; for details, see
Ma et al. (2000). Hence, the correlation coefficient curves can be adopted together with Fourier coefficients curves to characterise shape.

Moreover, a shape index is defined in Eq. 7, derived from the correlation coefficients measured at different azimuthal angles. This index is 1 for completely simultaneous fluctuations at all directions and lies between 1 and $N$ when fluctuations between certain azimuthal angles are correlated. In this study, $N$ is the number of sampling points (i.e., 64)

$$
\psi_{s i}=\frac{N / 2}{\sum_{m=1}^{N / 2} \rho_{m}}
$$

The diffraction shape index and the correlation coefficient appear to be rather insensitive to the number of particles in the measurement region (e.g., region of interest in image analysis), although an increasing number of particles will derogate the shape information (Ma et al., 2000). Hence, the best information can be obtained by allowing only one particle to be present at the same time in the measurement zone. This is the methodology underlying the image analysis in this study. For each type of aggregate, six particles are selected from Fig. 1 (for illustration purposes) and subjected to Fourier analysis. The calculation results are averaged over the six particles.

\section{APPLICATION TO TWO DIFFERENT TYPES OF AGGREGATE GRAINS}

\section{RESULTS}

Two types of aggregate, fluvial gravel and crushed rock, respectively, were investigated for illustration purposes in this study. For each type of aggregate, eighteen particles are selected and subjected to conventional image analysis, to stereological estimations, and to Fourier analysis. The selected particles are within a narrow size range around $18 \mathrm{~mm}$ (see Fig. 1) so as to eliminate the effect of size variations. The measurement results are averaged over the eighteen particles for each aggregate group. 


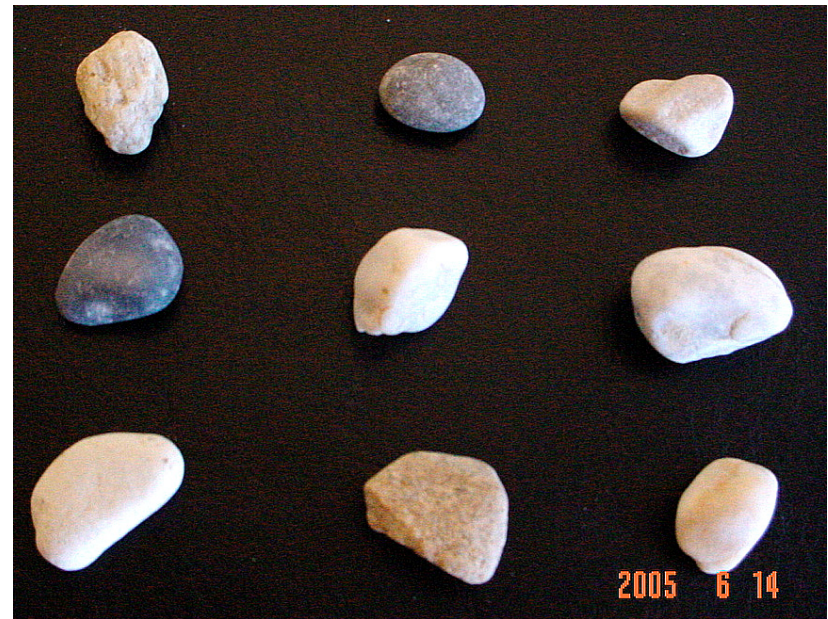

(a)

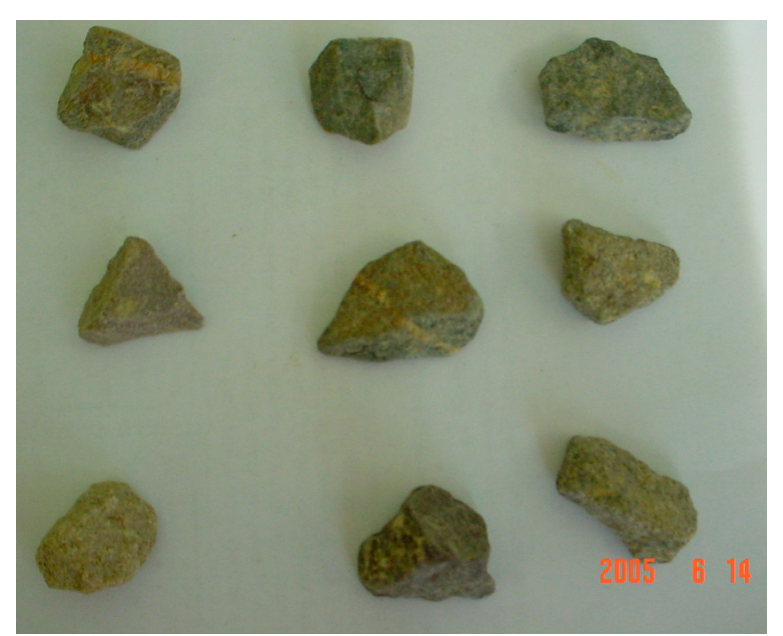

(b)

Fig. 1. Representative particles from fluvial gravel (a) and from crushed rock (b).

Table 2. Shape parameters obtained by quantitative analysis of projected images.

\begin{tabular}{l|l|l}
\hline Parameter & Fluvial gravel & Crushed rock \\
\hline Area $\left(\mathrm{cm}^{2}\right)$ & 2.427 & 2.823 \\
Perimeter $(\mathrm{cm})$ & 6.120 & 6.914 \\
Max Axis Length $(\mathrm{cm})$ & 2.067 & 2.293 \\
Breadth $(\mathrm{cm})$ & 1.563 & 1.812 \\
Arithmetic mean of Elongation ratio (Length/Breadth) & 1.330 & 1.281 \\
Weighted mean of Elongation ratio & 1.315 & 1.276 \\
Aspect ratio $\left(F_{\max } / F_{\min }\right)$ & 1.367 & 1.355 \\
Circularity $\left[\right.$ Perimeter $/\left(4 \pi^{*}\right.$ Area) $)$ & 1.257 & 1.359 \\
Rectangularity $($ Area/Area of bounding rectangle) & 0.730 & 0.682 \\
Fullness [Area/ $\left.\left(F_{\min }{ }^{*} F_{\max }\right)\right]$ & 0.733 & 0.712 \\
Flakiness ratio $\lambda($ thickness/breadth) & 0.393 & 0.325 \\
Sphericity ratio & 0.607 & 0.591 \\
Shape factor & 0.342 & 0.290 \\
\hline
\end{tabular}

The projection profiles are measured by automatic image analysis and the shape parameters are calculated (Table 2). The circularity is 1 for a circle in a plane (2D), and higher $(>1)$ for irregular shapes. The circularity of the particle projection profiles for the fluvial gravel (1.257) is lower than that of the crushed rock (1.359), revealing that the boundary of the fluvial gravel particles is more conforming to the ellipse type. The sphericity ratio is 1 for a sphere in space (3D), and lower $(<1)$ for irregular shape. The sphericity ratio for the fluvial gravel is slightly higher than that of the crushed rock, confirming that the latter aggregate type is more angular than the other.

Mora and Kwan (2000) proposed empirical equations correlating various shape parameters to the experimental measurements of angularity number. Table 3 shows the thus calculated results of the angularity number derived from different shape parameters. It is revealed that the flakiness ratio $(\lambda)$ and the shape factor can provide relatively reliable estimations of the angularity number, i.e., 6 and 10 respectively for the fluvial gravel and the crushed rock. However, sphericity ratio (reflecting similarity to sphere) is not sensitive enough to variations in fine structure of particle shape, since it does not contain sufficient shape information. The aggregate particles investigated in this study deal with a very narrow size range, thus the packing density is expected to be lower than in the case of a multi-size sample. As a consequence, the angularity number as calculated in Table 3 is expected to be higher than the actual angularity number of the multi-size aggregate sample of the same origin.

The stereological methods are also applied to the particles. In fact, the average tangent height $\bar{H}_{2}$ equals the Feret diameter averaged over all orientations. To simplify the procedures, $\bar{H}_{3}$ is averaged over thickness, length and breadth. Since size distribution 
of aggregate particles is not taken into consideration in this report, only near mono-size particles are analysed. This means that moments of the particle size distribution are simplified to

$$
M_{3}(d)=\left(\bar{H}_{3}\right)^{3} \quad M_{2}(d)=\left(\bar{H}_{3}\right)^{2} \quad M_{1}(d)=\bar{H}_{3} .
$$

This allows easy calculation of the shape indices $S_{1}{ }^{*}, S_{2}$ and $S_{3}$ (Table 4). However, it should be noted that the equations introduced here are readily applicable to more complicated cases of multi-size particle samples (e.g., coarse aggregate conforming to Fuller-type size distribution, or cement particles conforming to Rosin-Rammler size distribution).

Table 3. Angularity number calculated on the basis of various shape parameters (calculation according to the empirical equations available in Mora and Kwan (2000)).

\begin{tabular}{l|l|l}
\hline Parameter & Fluvial gravel & Crushed rock \\
\hline Flakiness ratio $\lambda$ & 5.93 & 10.93 \\
Sphericity ratio & 4.42 & 6.10 \\
Shape factor & 5.41 & 9.48 \\
\hline
\end{tabular}

The investigated two groups of aggregate particles are in the same size range, so the size parameters, such as the average tangent heights in 3D $\left(\bar{H}_{3}\right)$, and the specific surface area, are expected to be similar (see Table 4). The shape factors $S_{3}$ and $S_{2}$ are 0.524 [i.e., $V / M_{3}(d)=\left(\pi d^{3} / 6\right) / d^{3}$ ] and 3.14 [i.e., $\left.S / M_{2}(d)=\pi d^{2} / d^{2}\right]$, respectively for spheres. The closer the measurement to these values, the more the investigated particles approximate spheres. Table 4 reveals that the fluvial gravel comes closer to the spherical shape. This confirms that the coarse aggregates of fluvial origin can be reasonably replaced by spherical particles in computer simulation of particle packing in concrete (Stroeven and Stroeven, 1999); however, a simulation based on ellipsoids will surely provide better results. The ratio between the crushed rock and the fluvial gravel for $S_{1}^{*}, \quad S_{2}$ and $S_{3}$ is $0.92 \quad(=0.816 / 0.883), \quad 0.82$ $(=2.113 / 2.564)$ and $0.76(=0.431 / 0.566)$, respectively. The difference between the two aggregate types is increasing with the involved dimensions. This implies that a higher order shape parameter (in this case $S_{3}$ ) is more suitable for the purpose of distinguishing aggregate types in terms of shape, since it contains more shape information (in three dimensions) of the investigated particles. Hence, stereological approaches to shape characterization are superior to the conventional global shape parameters (Table 2).

Fig. 2 gives the signal intensity curves (polar coordinates in the azimuthal range of $0-2 \pi$, corresponding to the $x$-axis of index number) and Fourier curves for the fluvial gravel and the crushed rock. The curves are averaged over eighteen investigated particles for each group and normalised by the maximum value to eliminate the effect of size variations. It should be noted that the averaging procedures reduce the information of boundary structure variations; the impact of averaging will be more significant when the shape uniformity within the group of particles is of a relatively low level, as in the case of the crushed rock.

Table 4. Shape parameters obtained by stereological methods.

\begin{tabular}{l|l|l}
\hline Parameter & Fluvial gravel & Crushed rock \\
\hline Mean volume per particle $\bar{V}\left(\mathrm{~cm}^{3}\right)$ & 1.590 & 1.673 \\
Area of projection profile $\bar{A}\left(\mathrm{~cm}^{2}\right)$ & 2.427 & 2.823 \\
Perimeter of projection profile $\bar{L}_{P}(\mathrm{~cm})$ & 6.120 & 6.914 \\
Specific surface area $\bar{S} / \bar{V}\left(\mathrm{~cm}^{-1}\right)$ & 3.211 & 3.118 \\
Mean surface per particle $\bar{S}\left(\mathrm{~cm}^{2}\right)$ & 5.105 & 5.216 \\
$\bar{L}_{3}=4 \bar{V} / \bar{S}(\mathrm{~cm})$ & 1.246 & 1.283 \\
$\bar{H}_{3}(\mathrm{~cm})$ & 1.411 & 1.571 \\
$\bar{H}_{2}(\mathrm{~cm})$ & 1.814 & 2.039 \\
$S_{S u r f a c e}$ curvature $\bar{K}_{S}\left(\mathrm{~cm}^{-1}\right)$ & 0.866 & 0.770 \\
$S_{3}$ & 0.566 & 0.431 \\
$S_{2}$ & 2.564 & 2.113 \\
$S_{1}^{*}$ & 0.883 & 0.816 \\
\hline
\end{tabular}




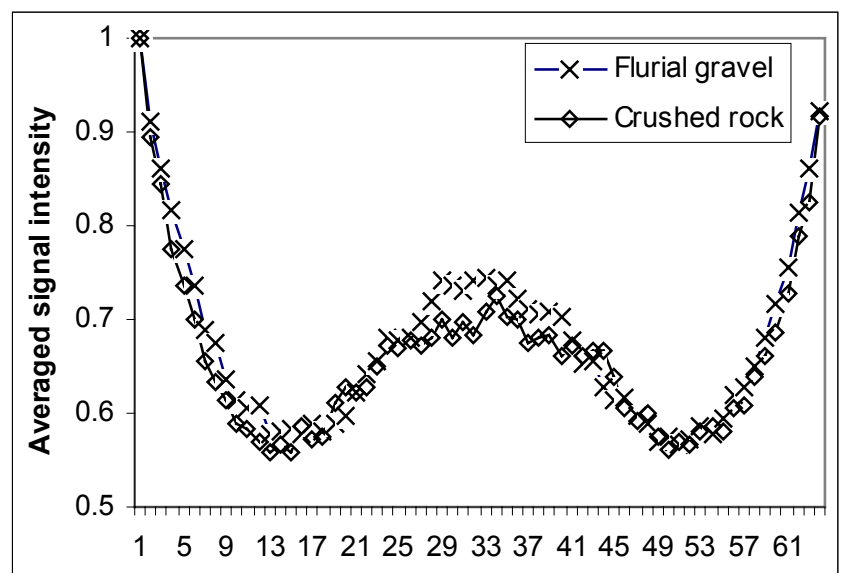

(a)

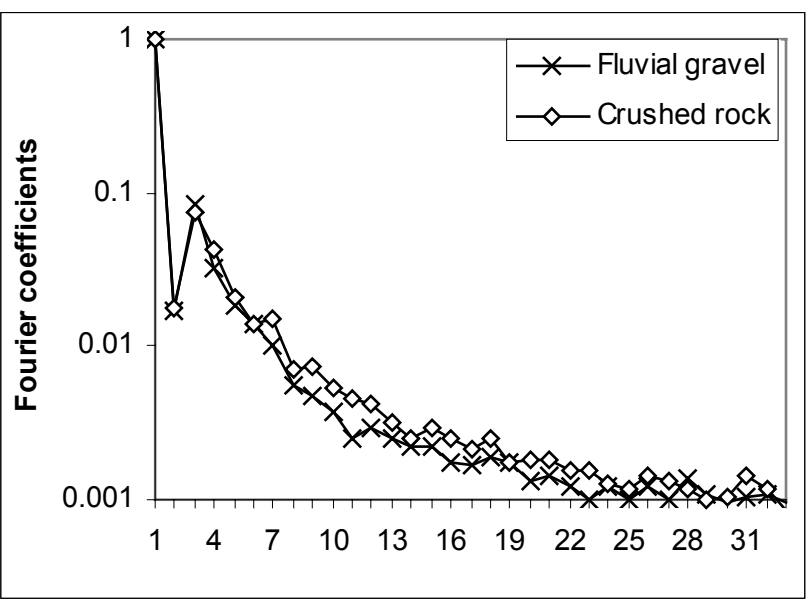

(b)

Fig. 2. Averaged signal intensity patterns (a), normalised by the respective maximum value, and Fourier coefficient curves (b) for the fluvial gravel and for the crushed rock.

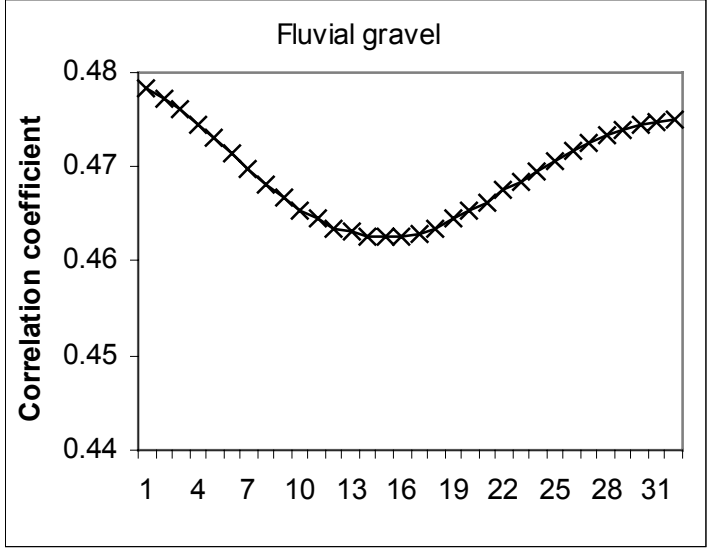

(a)

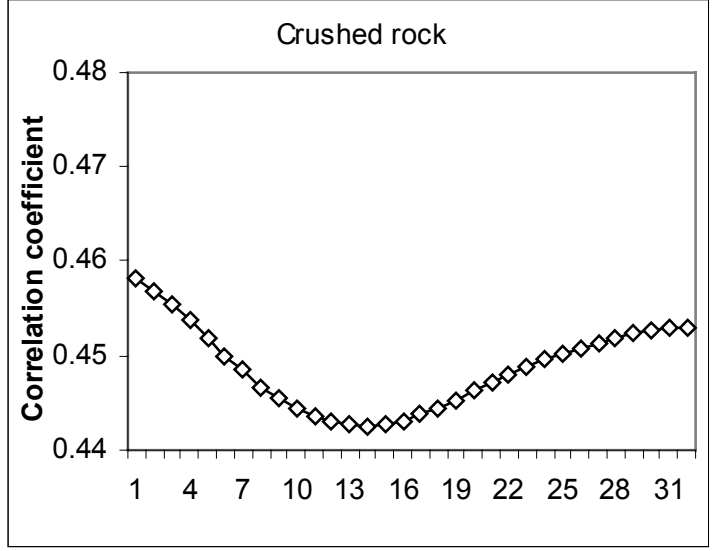

(b)

Fig. 3. Correlation coefficient curves for the investigated two types of coarse aggregate. The curves are averaged over eighteen particles for each group of aggregate.

Table 5. Shape parameters obtained by Fourier analysis.

\begin{tabular}{l|l|l}
\hline Parameter & Fluvial gravel & Crushed rock \\
\hline Lumpiness $(L)$ & 0.187 & 0.201 \\
Roughness $(R)$ & 0.021 & 0.030 \\
Texture $(T)$ & 0.011 & 0.014 \\
$A_{2} / A_{4}$ & 5.315 & 6.011 \\
$A_{3} / A_{4}$ & 2.077 & 3.707 \\
Shape index $\psi_{s i}$ & 1.961 & 2.486 \\
Circular index & 0.678 & 0.662 \\
Estimated packing density (-) & 0.55 & 0.49 \\
Experimental packing density $(-)$ & 0.56 & 0.52 \\
Optimal volume fraction of fine aggregate & 0.25 & 0.30 \\
\hline
\end{tabular}


Fig. 3 presents the averaged correlation coefficient curves for the two aggregate types. It could be expected that the non-uniformity in shape of the crushed rock particles would be suppressed in the averaging procedures. The correlation coefficients $\rho_{m}$ for the crushed rock are lower than for the fluvial gravel (Fig. 3), implying that the boundaries of the aggregate particles are varying to a more significant extent in the case of the crushed rock (even after the averaging procedures). The averaged shape index is 1.96 and 2.48 (Table 5), respectively, for the fluvial gravel and for the crushed rock. Moreover, compared to the fluvial gravel, the crushed rock covers a much wider range of shape indices (from 1.8 to 3.2), indicating a lower shape uniformity within the group. It is interesting to see that the ratio of average $\psi_{s i}$ values of the fluvial gravel and the crushed rock is $0.78(=1.96 / 2.48)$, which is close to the ratio of $S_{3}$ values $(0.76)$ obtained by the stereological methods. This indicates that although based on $2 \mathrm{D}$ projection images, the Fourier analysis is able to capture shape information of the fine structure of aggregate particles. The differences between the two aggregate types will be more significant when the number of sampling points (in this case 64 ) on each projection profile is further increased to 128 or 256.

Table 5 lists the averaged results of shape parameters obtained from Fourier analysis. Fig. 4 presents the Fourier coefficients $A_{2}$ and $A_{3}$ as a function of $A_{4}$, indicating a higher degree of shape uniformity in the group of the fluvial gravel than in

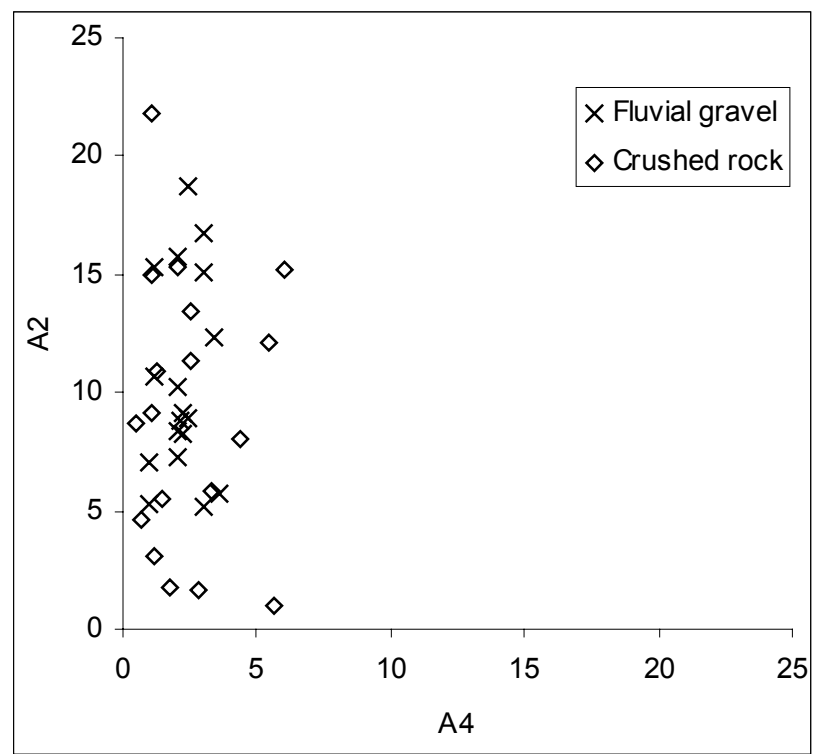

(a) that of the crushed rock. The close values of $A_{2} / A_{4}$ are expected in view of the moderate differences between the two aggregate types in terms of global structures. Table 5 reveals that the ratio of $A_{3} / A_{4}$ is relatively high in the case of the crushed rock. A higher value of $A_{3} / A_{4}$ is corresponding to a larger similarity to a triangle; hence, the projection profiles of the crushed rock particles closer approximate a triangular shape, a conclusion confirmed by visual observation of the particles. The lumpiness of the crushed rock is slightly higher than that of the fluvial gravel, as a result of moderate differences between the two aggregate types. However, the roughness of the crushed rock is exceeding that of the fluvial gravel by $43 \%$ (quite a dramatic difference).

Gotoh et al. (1985) proposed an empirical equation to correlate a specific shape parameter and packing density for a group of identical particles (i.e., no variations in size and shape within the group). Their study involved various types of shape, including ellipsoid, rectangular parallelepiped, and cylinder. The shape parameter $A$ defined in Gotoh et al. (1985) is expressed by $R S / V$, where $R$ is particle radius averaged over all orientations; $S$ and $V$ are the surface area and volume of a single particle. The thus-defined shape parameter is 3 for spherical particles. According to ASTM, a packing density $\phi$ of 0.67 is assumed for mono-size spherical particles, allowing estimation of the packing density for an arbitrary type of aggregate.

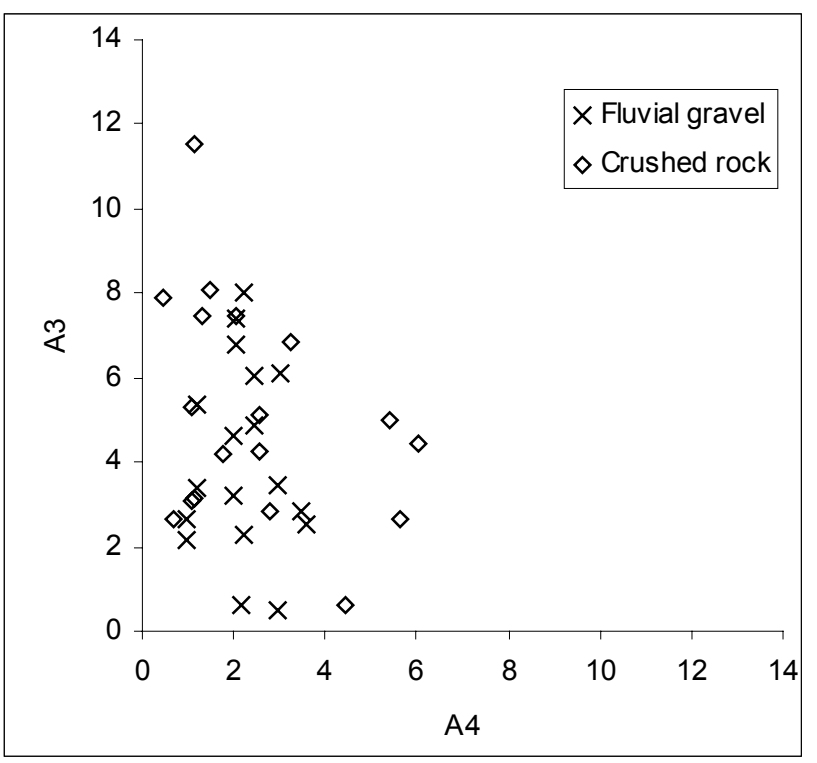

(b)

Fig. 4. Fourier coefficients $A_{2}(a)$ and $A_{3}$ (b) plotted versus $A_{4}$ for the investigated aggregate types. The fluvial gravel is much more uniform in particle shape than the crushed rock. 
The afore-mentioned stereological parameters can be used in this study to estimate this shape parameter. As a consequence, $R$ is replaced by $\bar{H}_{3} / 2, S$ and $V$ are replaced by the average values $(\bar{S}$ and $\bar{V}$, respectively), leading to the equation of $\bar{H}_{3} \bar{S} /(2 \bar{V})$. The calculation yields similar values of shape parameters for the two groups, which are expected in view of the quite close values of the involved parameters (see Table 4). Hence, global parameters for size characterisations (such as $\bar{H}_{3}, \bar{S}$ and $\bar{V}$ ) cannot be combined in this way to represent shape characteristics, particularly in the case of irregular shape.

Thus, in this study, the shape index $\psi_{\mathrm{si}}$ obtained by Fourier analysis is used to represent the overall shape characteristics of the investigated aggregates. Since the shape index $\psi_{\mathrm{si}}$ equals 1 for spheres, whereas the shape parameter $A$ is 3 for spheres in Gotoh et al. (1985), the shape index values in Table 5 are adapted by multiplying with 3 . This yields 5.8 and 7.4 respectively for the fluvial gravel and the crushed rock. Hence, the calculated packing density is 0.55 and 0.49 , respectively, for the two types of aggregate. Hughes and Bahramian (1966) experimentally measured the angularity factor (defined as the packing density ratio of spherical glass marbles and the investigated aggregate) for different types of crushed rock (e.g., limestone, basalt and granite) and for rounded fluvial gravel. The angularity factor reported by the authors is varying between 1.28 and 1.295 for the crushed rocks, and 1.206 for the fluvial gravel, provided that the aggregate particles are in a narrow size range (thus considered as mono-size) around 18 $\mathrm{mm}$. ASTM assumes the maximum packing density for mono-size spherical particles with smooth surface is 0.67 . Hence, the packing density (volume fraction of aggregate) is estimated to be about 0.56 for rounded gravel and 0.52 for the crushed rock.

In conclusion, the shape index obtained by Fourier analysis can effectively distinguish aggregate types with various shape characteristics. Based on this parameter, the packing density can be estimated, which has been demonstrated to yield comparable results with the experimental measurements of packing density according to ASTM. When applied to aggregate particles covering different size ranges, the packing density for each size fraction can be determined separately and then averaged on the basis of weight of each size fraction to yield overall packing density of the aggregate.

\section{DISCUSSION: IMPLICATIONS FOR CONCRETE PERFORMANCE}

The shape parameters derived from stereological methods and Fourier analysis could provide implications for concrete mix proportioning with the objective of property optimisation. On the basis of 45 concrete mixes, Jamkar and Rao (2004) studied the potential of IAPST of coarse aggregate as a parameter for concrete mix proportioning. It can be expected that for the same compaction factor and water cement ratio, the water content will increase in the case of an aggregate with higher roughness $(R)$ and texture $(T)$. Relevant to the investigated aggregates in this study, the optimal volume fraction of fine aggregate (sand) should be increased from $25 \%$ to $30 \%$ when the coarse aggregate of fluvial gravel is replaced by the crushed rock (Table 5). The obvious influences of shape and texture characteristics of coarse aggregate on water content, volume fraction of fine aggregate (sand), and compaction factor indicate that aggregate shape should be incorporated as an important technical parameter in the design of concrete mix proportioning.

As mentioned earlier, shape and texture of coarse aggregate exert significant effects on mechanical and transport properties of concrete. This can positively influence durability potentialities of the composite. A significant advantage of the concurrent algorithmbased SPACE (Stroeven, 1999) simulation system (over common Random Sequential Addition-based systems) is its capability to properly simulate configuration aspects of dense random packing of particulate material structure, and - thereby - to allow studying structure-sensitive properties of cementitious materials. Moreover, realistic packing densities for binder and aggregates grains in concrete (up to about 0.76 ) can be realized. With the HADEX toolbox (new generation concurrent algorithm-based computer simulation system, which is now being developed), the effect of shape characteristics of aggregate can be incorporated in the simulation of material structure. On the basis of the relationship between aggregate shape and material behaviour (obtained by experimental testing), a simple modelling concept can be developed correlating mechanical performance characteristics to the shape parameters. This would allow qualifying effects of aggregate shape on concrete performance in structural terms, thereby promoting the design of concrete materials. Extending the HADES toolbox capabilities to hydrated systems will lead to an integrated concept. 


\section{CONCLUSIONS}

This paper presents a reliable and economic methodology for shape characterisation by means of stereological methods and Fourier analysis, and illustrates the application of these methods to two types of coarse aggregates of different origins. Stereological estimations render possible deriving threedimensional shape information of the aggregate from two-dimensional projection profiles of the particles, on the basis of an economical and proper sampling strategy. Thus, stereological shape indices, incorporating higher order (i.e., three-dimensional) shape parameters, are more suitable than the conventional two-dimensional global shape parameters for distinguishing among different aggregate types.

The research also demonstrates that more efficient shape parameters (compared to conventional roundness, circularity, sphericity and etc.) can be derived from Fourier analysis to quantitatively characterise the global as well as the fine structure of shape characteristics, encompassing lumpiness, roughness and texture of the aggregate grains. These parameters render possible distinguishing the types of aggregates in an economic, efficient and reliable way. The shape information derived from Fourier analysis can provide useful implications for concrete production (e.g., concrete proportioning) and for concrete performance as well.

\section{REFERENCES}

ASTM D 3398-97 (1997). Standard Test for Index of Aggregate Particle Shape and Texture. West Conshohocken, USA

Beddow JK (1985). Recent applications of morphological analysis. In: Beddow JK, ed, Particle Characterization in Technology II (Morphological Analysis). Boca Raton: CRC Press, 149-71.

BS 812 Part-1 (1975). Methods for determination of particle size and shape, British Standards, UK.

Bouman ET, Saga K, Drummond TW (2000). Particle shape characterisation using Fourier Analysis, CUED/DSoils, TR315, 1-20.
Gonzales R, Woods R (1992). Digital Image Processing. Addison-Wesley Publ Co, 81-125.

Gotoh K, Kumamoto M, Meloy TP (1985). Particle shape characterisation from packing density. In: Beddow JK, ed. Particle Characterization in Technology II (Morphological Analysis). Boca Raton: CRC Press, 15-22.

Huang EY (1962). A test for evaluating the geometric characteristics of coarse aggregate particles. ASTM Proc 62:1223-42.

Hughes BP, Bahramian B (1966). A laboratory test for determining the angularity of aggregate, Mag Concr Res 18;56:147-52.

Jamkar SS, Rao CBK (2004). Index of Aggregate Particle Shape and Texture of coarse aggregate as a parameter for concrete mix proportioning. Cem Concr Res 34:2021-7.

Kwan AKH, Mora CF, Chan HC (1999). Particle shape analysis of coarse aggregate using digital image processing. Cem Concr Res 29:1403-10.

Ma Z, Merkus HG, de Smet JGAE, Heffels C, Scarlett B (2000). New developments in particle characterization by laser diffraction: size and shape. Powd Techn 111: 66-78.

Mora CF, Kwan AKH (2000). Sphericity, shape factor, and convexity measurement of coarse aggregate for concrete using digital image processing. Cem Concr Res 30:351-8.

Shergold FA (1953). The percentage voids in compacted gravel as a measure of its angularity. Mag Concr Res 13/5:3-10.

Stroeven M (1999). Discrete Numerical Modelling of Composite Materials - Application to Cementitious Materials. PhD Thesis, Delft University Press, Delft.

Stroeven P, Stroeven M (1999). Assessment of packing characteristics by computer simulation. Cem Concr Res 29:1201-6.

Underwood EE (1970). Quantitative Stereology. AddisonWesley Publ Co., Reading (Ma).

Underwood EE (1976). Three-dimensional shape parameters from planar sections, NBS Spec Publ 431, Fourth Int Congr Stereol. US Printing Off: Washington, 91-2.

Yamamoto H, Matsuyama T, Wada M (2002). Shape distinction of particulate materials by laser diffraction pattern analysis. Powd Techn 122:205-11. 